\title{
Vibronic excitations of large molecules in solution studied by two-color pump-probe experiments on the 20 fs time scale
}

\author{
S. H. Ashworth, T. Hasche, M. Woerner, E. Riedle, and T. Elsaesser \\ Max-Born-Institut für Nichtlineare Optik und Kurzzeitspektroskopie, Rudower Chaussee 6, D-12489 Berlin, \\ Germany
}

(Received 21 December 1995; accepted 8 January 1996)

\begin{abstract}
The ultrafast vibronic response of organic dye molecules in solution is studied in pump-probe experiments with 30 fs excitation pulses resonant to $S_{0}-S_{n}$ transitions. The molecular dynamics is probed either by pulses at the same spectral position or by $20 \mathrm{fs}$ pulses overlapping with both the $S_{0}-S_{1}$ absorption and emission bands. Three contributions on distinctly different time scales are observed in the temporally and spectrally resolved two-color measurements. In the regime below 50 fs, a strong coherent coupling of the $S_{0}-S_{n}$ and the $S_{0}-S_{1}$ transitions occurs that is due to coherent vibrational motions in the electronic ground state. This signal is superimposed on the fast bleaching of the electronic ground state, resulting in a steplike increase of transmission. In the range of the $S_{0}-S_{1}$ emission band, one finds a subsequent picosecond rise of transmission that is due to stimulated emission from vibronic $S_{1}$ states. The data demonstrate that the relaxation of $S_{n}$ states directly populated by the pump pulses is much faster than the buildup of stimulated emission. This gives insight into different steps of intramolecular vibronic redistribution and is compared to the $S_{n}-S_{1}$ relaxation in other molecules. (C) 1996 American Institute of Physics.
\end{abstract}

[S0021-9606(96)02814-2]

\section{INTRODUCTION}

Elementary excitations of large molecules in liquids show ultrafast relaxation dynamics that strongly influence the electronic and vibrational spectra of the molecules. Different phenomena have been distinguished and characterized in optical experiments: Optical excitation with coherent light induces a coherent optical polarization of the molecules that decays by phase-breaking interactions with the surrounding. ${ }^{1-10}$ For vibronic excitations, phase relaxation times in the order of 10 to 60 fs have been measured by a variety of techniques like time-resolved photon-echo spectroscopy ${ }^{3-7}$ or hole burning. ${ }^{2}$ In the simplest approach, this behavior has been described by the optical Bloch equations introducing a phenomenological phase relaxation time $T_{2}$. More sophisticated models were used to interpret the experimental results in terms of the solute-solvent interaction, i.e., a variation of the vibronic transition frequency by fluctuations in the bath, and have taken into account the non-Markovian character of the bath response. ${ }^{6,7}$ For vibrational excitations, a considerably slower decay of phase coherence was found, corresponding to phase relaxation times of $0.5 \mathrm{ps}$ up to several picoseconds. $8,9,11$

The redistribution of vibronic and vibrational populations represents a second class of relaxation phenomena that are important for the intramolecular equilibration of population and for the transfer of energy from the solute to the solvent. ${ }^{12}$ In the following, we concentrate on the relaxation in electronically excited states. Vibronic relaxation was mainly studied in the first excited singlet state and occurs in most cases on a time scale well below 1 ps. ${ }^{1,12-14}$ Redistribution within the large manifold of states leads to an irreversible transfer of population by internal conversion from states at higher energies to those close to the purely electronic $S_{1}$ state.

This behavior was also found after excitation of high lying singlet states $S_{n}$ and is the basis of an empirical rule formulated by Kasha stating that fluorescence originates from the first excited singlet state. ${ }^{15}$ A closer look shows that $S_{n}-S_{1}$ relaxation implies different processes. First, there is a transfer of population from the optically coupled subset of states to the dense manifold of other, nearly isoenergetic, vibronic states which belong either to the same or to other electronic states. The interaction between these two groups of levels has been attributed to nonadiabatic coupling terms in the molecular Hamiltonian. In the so-called statistical limit, nonradiative transitions rates in large molecules have been calculated from Fermi's golden rule. ${ }^{16}$ After the transfer to this intramolecular bath, redistribution processes within the vibronic manifold that include crossing of electronic potential surfaces, lead to a rapid spreading of population over many levels and-finally - to the accumulation at the bottom of the $S_{1}$ state. A theoretical description of the latter step is very difficult for large systems because of the high number of degrees of freedom involved in the overall relaxation.

Internal conversion from high-lying singlet states has been studied in a few experiments on a time scale of 100 fs. ${ }^{17-19}$ In some cases, upper limits for the redistribution times have been estimated from the pulse-limited response of the molecules. ${ }^{17}$ For rhodamine and oxazine dyes, overall redistribution times of about $200 \mathrm{fs}$ were resolved in twocolor pump-probe studies, monitoring the onset of stimulated emission from $S_{1} \cdot{ }^{18,19}$ However, a separation of the different steps occurring along the relaxation pathway, in particular during and immediately after excitation was not possible because of the limited time resolution. In addition, a 
detailed theoretical description of such processes allowing a quantitative analysis of the experiments is not yet available. To get better insight into such phenomena, experiments with higher time resolution are required which probe both the initially excited manifold of states as well as the final levels in the range of the electronic $S_{1}$ state.

Very recently, we have reported the first two-color pump-probe study of highly excited dye molecules in solution with a time resolution of $20 \mathrm{fs} .{ }^{20}$ Coherent interaction of polarizations on vibronic $S_{0}-S_{n}$ and $S_{0}-S_{1}$ transitions was observed and attributed to a coupling via coherent vibrational motion in the electronic ground state. Molecules excited to $S_{n}$ levels showed a very fast bleaching of the ground state and a slower picosecond accumulation at the bottom of the $S_{1}$ state, resulting in a delayed rise of stimulated emission. In this paper, we present new data on both the coherent response and the latter step of radiationless $S_{n}-S_{1}$ relaxation. Two-color pump-probe studies with 20 fs time resolution were performed in wavelength ranges around 425 and around $850 \mathrm{~nm}$. The decay of the initially excited $S_{n}$ states and the buildup of the emission spectrum was monitored in spectrally and temporally resolved experiments. The data give evidence that the time evolution of initially excited vibronic $S_{n}$ states occurs in less than $50 \mathrm{fs}$ which is much faster than the accumulation of molecules at the bottom of $S_{1}$ that proceeds with time constants of about $1 \mathrm{ps}$. These results allow us to distinguish different processes of ultrafast vibronic relaxation.

The paper is organized as follows. After a brief description of the experimental techniques in Sec. II, we present results of the different pump-probe experiments (Sec. III). The interpretation of our data is discussed in Sec. IV, including a comparison to other work on the ultrafast response of large molecules in solution. Finally, we give a brief summary in Sec. V.

\section{EXPERIMENTAL TECHNIQUES}

The pump and probe pulses used in our experiments are derived from a self-mode-locked Ti:sapphire laser pumped by a continuous-wave argon ion laser. The mode-locked oscillator with a cavity design similar to Refs. 21 and 22 provides trains of $20 \mathrm{fs}$ pulses centered at $850 \mathrm{~nm}$ with a repetition rate of about $90 \mathrm{MHz}$ and an average power of up to $800 \mathrm{~mW}$ (pump power $8 \mathrm{~W}$ ). The spectral profile of these pulses is plotted in Fig. 1.

Excitation pulses at $425 \mathrm{~nm}$ are generated by frequency doubling the main fraction of the laser output. ${ }^{23}$ After passing the output coupler of the laser, the fundamental pulse train is recompressed and focused with a $f=75 \mathrm{~mm}$ lens onto a $400 \mu \mathrm{m}$ thick BBO crystal. The second harmonic with an average power of up to $40 \mathrm{~mW}$ is recollimated with a $f=50$ $\mathrm{mm}$ lens and travels through a second prism compressor. After recompression, the pulse duration was 30 fs. The spectrum of the blue pulses is shown in Fig. 1, the bandwidth (FWHM) has a value of $11 \mathrm{~nm}$.

In the pump-probe experiments, either a small fraction of the fundamental laser output or a weak part of the second

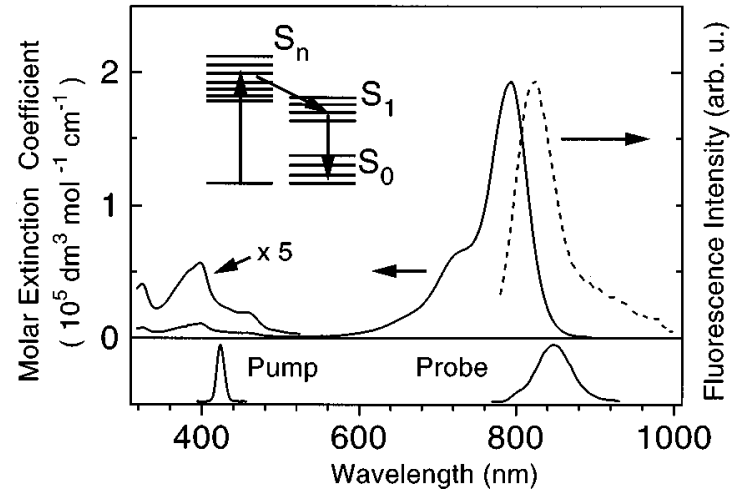

FIG. 1. Absorption (solid line) and fluorescence spectra (dashed line) of the dye IR 125 dissolved in ethylene glycol. The molar extinction coefficient and the fluorescence intensity are plotted as a function of wavelength. The spectra show a small Stokes shift of about $430 \mathrm{~cm}^{-1}$ and an approximate mirror symmetry. The spectral profiles of the $30 \mathrm{fs}$ excitation pulses centered at $425 \mathrm{~nm}$ and the $20 \mathrm{fs}$ probe pulses at $850 \mathrm{~nm}$ are shown on the abscissa. The insert gives a schematic energy level diagram of the molecules.

harmonic serve as probe pulses. In the two-color experiments, pump and probe beams travel collinearly and are focused with a $f=75 \mathrm{~mm}$ lens onto the sample, resulting in a spot size of about $30 \mu \mathrm{m}$. In the measurements with probe pulses at $425 \mathrm{~nm}$, pump and probe beam subtend a small angle of about $5 \mathrm{deg}$. In the different experiments, the ratio of pump to probe intensity was between 100:1 and 1000:1 and the polarization of pump and probe was parallel. Both pulses are precompensated in compressors made of fused silica prisms in order to achieve minimal pulse length at the sample. The pulse lengths were routinely monitored by autoand cross-correlation measurements at the sample position with $100 \mu \mathrm{m}$ thick KDP and BBO crystals, respectively.

After separation from the pump light, the transmitted infrared probe beam is passed through a $f=250 \mathrm{~mm}$ monochromator and detected by a silicon photodiode either integrally (zeroth order of the monochromator) or spectrally resolved with a bandwidth of $10 \mathrm{~nm}$. The blue probe beam is directly monitored with a photomultiplier. To obtain a high signal-to-background ratio, the pump beam is modulated by a mechanical chopper and the transmission changes of the probe are measured with a lock-in amplifier.

Commercially available IR125 was dissolved in ethylene glycol with a concentration of $5 \times 10^{-4} \mathrm{M}$. A free jet of the dye solution with a thickness of $70 \mu \mathrm{m}$ was used in order to minimize thermal effects in the sample. The maximum absorbance of the sample was 0.6 (at the $S_{0}-S_{1}$ absorption maximum), resulting in negligible reshaping of the spectra of pump and probe pulses, i.e., propagation effects due to changes of the pulse spectra are not relevant in our experiments. In the measurements, a small fraction of the molecules (about $10^{-3}$ ) in the irradiated volume of the sample are excited to $S_{n}$ states by each individual pulse. For this weak excitation, cumulative pump-probe signals which are caused by the combined interaction of successive pump pulses (separation $11 \mathrm{~ns}$ ) with the sample, can be neglected. This conclusion is confirmed by measurements with different 


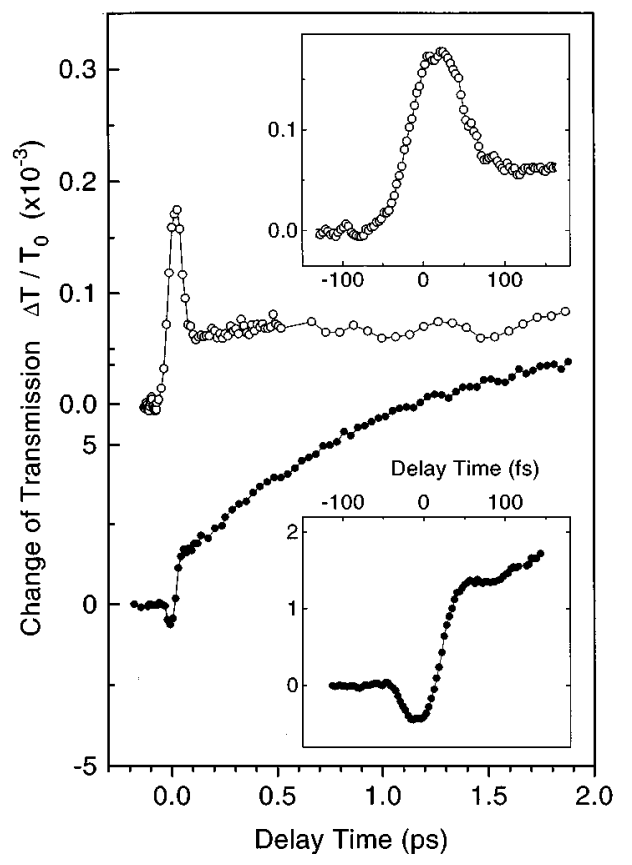

FIG. 2. Time resolved pump-probe data measured with $30 \mathrm{fs}$ excitation at $425 \mathrm{~nm}$. The change of transmission $\Delta T / T_{0}=\left(T-T_{0}\right) / T_{0}$ is shown as a function of delay time $\left(T, T_{0}\right.$ : transmission of the sample with and without excitation). The upper trace (open circles) was recorded with probe pulses at $425 \mathrm{~nm}$. The lower transient (solid circles) was measured with the spectrally integrated probe at $850 \mathrm{~nm}$. In the inserts, the initial parts of both curves are plotted on an expanded time scale.

flow speeds of the dye jet, i.e., exchange rates of sample volume that give identical results.

\section{EXPERIMENTAL RESULTS}

The absorption and emission spectra of IR125 dissolved in ethylene glycol are displayed in Fig. 1 . The $S_{0}-S_{1}$ absorption band and the $S_{1}-S_{0}$ emission band show a relatively small Stokes shift of $430 \mathrm{~cm}^{-1}$ and are close to mirror symmetry. This behavior differs significantly from other dye molecules emitting in the near-infrared, e.g., styryles, and points to a relatively rigid molecular structure in which the modes coupling to the $S_{0}-S_{1}$ transition undergo minor changes of their frequency or Franck-Condon factor upon electronic excitation to the $S_{1}$ state. The $S_{0}-S_{1}$ transition shows a very high molar extinction coefficient (maximum value $2 \times 10^{5} \mathrm{dm}^{3} \mathrm{~mol}^{-1} \mathrm{~cm}^{-1}$ ) whereas the $S_{0}-S_{n}$ transitions pumped by the excitation pulses in the time-resolved experiments are considerably weaker.

In the following, we present data measured with probe pulses at $\lambda_{\mathrm{pr}}=425$ or $850 \mathrm{~nm}$. In the latter case, the full probe spectrum overlaps with both the absorption and emission of the $S_{0}-S_{1}$ transition. In Fig. 2, the time resolved transmission changes $\Delta T / T_{0}=\left(T-T_{0}\right) / T_{0}$ measured with spectrally integrated probe pulses are plotted as a function of time delay between pump and probe $\left(T, T_{0}\right.$ : transmission of the sample with and without excitation). The data recorded at $\lambda_{\mathrm{pr}}=425 \mathrm{~nm}$ (upper trace: open circles) show a very short spike around delay zero which is superimposed onto a step-

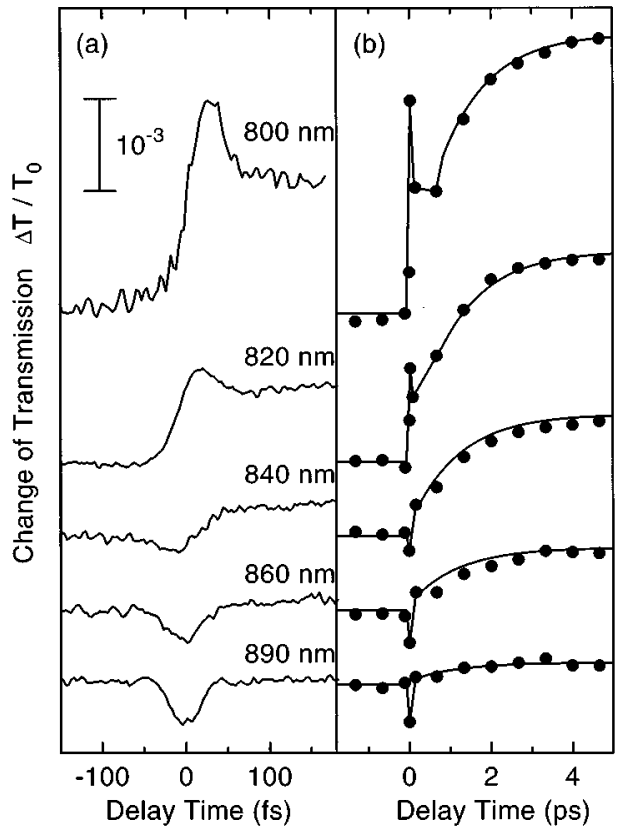

FIG. 3. Spectrally and temporally resolved transmission changes plotted on a (a) femtosecond and (b) picosecond time scale (excitation wavelength 425 $\mathrm{nm}$ ). The probe pulses centered at $850 \mathrm{~nm}$ were spectrally dispersed after interaction with the sample (detection bandwidth $10 \mathrm{~nm}$ ). The transients in (a) show a coherent component at early times and a steplike increase of transmission in the range of the $S_{0}-S_{1}$ absorption band. The picosecond rise in (b) follows a kinetics with a common rise time of $1.2 \mathrm{ps}$ (solid lines) at the different spectral positions and is due to increasing stimulated emission from the $S_{1}$ state.

like increase of transmission extending well into the picosecond regime. In the upper inset, the signal during the first 200 fs is plotted on an expanded scale, demonstrating that the fast transmission peak is essentially pulse limited. It is observed in measurements with parallel polarization of pump and probe pulses and decreases significantly for orthogonal polarization. This finding points to the coherent character of this contribution which is related to the third order nonlinearity of the dye molecules.

The second transient in the lower part of Fig. 2 (solid circles) was measured with a spectrally integrated probe pulses centered at $850 \mathrm{~nm}$. After a very fast initial rise of the signal that occurs on a similar time scale as the coherent spike in the $425 \mathrm{~nm}$ data, a much slower increase of transmission is observed. The signal reaches a maximum after about $3 \mathrm{ps}$ and decays subsequently with the lifetime of the $S_{1}$ state of $460 \mathrm{ps}$. This latter value was measured in an independent experiment with picosecond excitation of the molecules at $684 \mathrm{~nm}$ and streak camera detection of the resulting fluorescence. It should be noted that the picosecond rise of the signal is absent in the time-resolved data taken at $425 \mathrm{~nm}$.

Much better insight into the different contributions to the transmission signal is gained by spectrally resolving the probe pulses in the two-color experiment. In Fig. 3, the timeresolved response is plotted for $10 \mathrm{~nm}$ wide spectral components of the probe between 800 and $890 \mathrm{~nm}$. The data for the first 200 fs [Fig. 3(a)] exhibit a fast coherent contribution, 


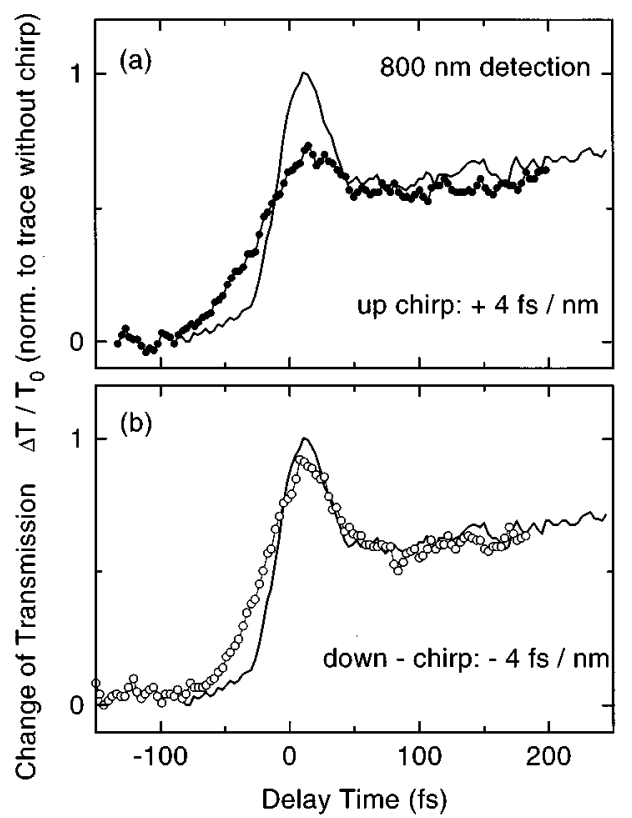

FIG. 4. Temporally and spectrally resolved data at a probe wavelength of $800 \mathrm{~nm}$ measured with chirped and unchirped excitation pulses at $425 \mathrm{~nm}$. The linear positive chirp, i.e., increasing frequency with time [solid circles in (a)], and negative chirp [open circles in (b)] of the pump pulses was introduced by changing the alignment of a prism compressor. In both cases, a broadening of the signal rise observed with unchirped pulses (solid lines) is found which is due to the associated pulse lengthening. The peaklike structure around delay zero is more pronounced for pump pulses with negative than with positive chirp.

the shape of which depends on the specific spectral position, and - at the shorter wavelengths - a steplike increase of transmission. The coherent component is related to a Ramantype excitation of the electronic ground state ${ }^{20}$ as will be discussed below, whereas the steplike transmission change is due to the (incoherent) bleaching of the electronic ground state.

Substantial changes of the coherent signal occur when a finite frequency chirp of the pump pulse is introduced. In Fig. 4, we present spectrally resolved pump-probe data at $\lambda_{\mathrm{pr}}=800 \mathrm{~nm}$ for pump pulses with positive chirp, i.e., increasing frequency with time [solid circles in Figs. 4(a), and 4(b)] negative chirp [open circles in Fig. 4(b)] compared to data without chirp [solid lines in Figs. 4(a) and (b)]. The desired linear chirp of the pump pulses has been introduced by changing the alignment of a prism compressor which had been calibrated by introducing defined pieces of fused silica glass into the optical path. With negligible chirp, one observes a sharp peaklike structure of positive sign. After chirping the pump pulses, a broadening of the femtosecond rise is found for both (a) up and (b) down chirp which is due to the pulse lengthening by chirping. In addition, we observe a weakening of the peaklike structure around delay zero which is more pronounced for the positive chirp [solid circles in Fig. 4(a)] than for the negative chirp [open circles in Fig. 4(b)].

At the spectral edges of the probe pulses, the transient data exhibit an oscillatory behavior on a time scale much

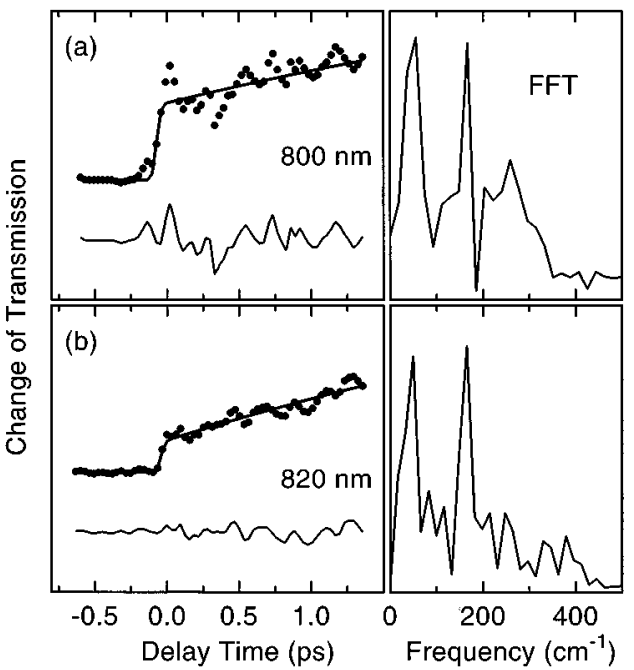

FIG. 5. Spectrally dispersed transient data at the short wavelength edge of the probe pulses. Both at (a) $800 \mathrm{~nm}$ and (b) $820 \mathrm{~nm}$ an oscillatory behavior is observed on a time scale outside pump-probe coincidence. On the left hand side, the time-resolved data are shown as solid circles. The solid line is a simulation of the steplike increase at early times and the picosecond rise of the signal. The downwards shifted traces (solid lines) represent the data minus simulation curves [cf. Fig. 3(b)]. The corresponding Fourier transform plots (FFT) of the data-minus-simulation traces are shown on the righthand side.

longer than the cross-correlation width. Spectrally dispersed data at (a) $800 \mathrm{~nm}$ and (b) $820 \mathrm{~nm}$ are shown in Fig. 5. To emphasize these oscillations we subtracted the calculated solid lines in Fig. 5 from our time-resolved data (solid circles). The calculation, which is based on a rate equation model, simulates the incoherent molecular response by a first instantaneous rise of transmission and a consequent delayed component with a rise time of $1.2 \mathrm{ps}$. The lower traces (solid lines) on the left hand side of Figs. 5(a) and 5(b) represent the extracted oscillations. The Fourier transforms of these time traces are plotted on the right hand side of Fig. 5. At the short-wave edge of the probe pulses [Figs. 5(a) $800 \mathrm{~nm}$ and 5 (b) $820 \mathrm{~nm}$ ] one recognizes a similar pattern in the low frequency part of the Fourier spectra $\left(0\right.$ to $\left.200 \mathrm{~cm}^{-1}\right)$. As will be discussed below, these oscillations are due to a coherent vibrational motion in the electronic ground state of the molecule caused by a Raman-type excitation by the pump pulses.

The spectrally resolved data taken for picosecond delay times [Fig. 3(b)] show the same slow rise of transmission as the spectrally integrated measurement in Fig. 2. Within the experimental accuracy, the rise occurs with the same time constant of $1.2 \mathrm{ps}$ (solid lines: monoexponential fits) at the different wavelengths. From the full set of time-resolved data, we derived the transient spectra presented in Figs. 6(a) and 6(b) (symbols). The fast bleaching component at a delay time of $80 \mathrm{fs}$ is shown in Fig. 6(a). The transient gain spectra [shown in Fig. 6(b)] were calculated from the amplitudes of the overall transmission change at a fixed delay time by subtracting the bleaching component observed at $80 \mathrm{fs}$. The gain spectra are normalized to the maximum signal observed after 5 ps. Solid lines are included for each delay time as a guide 


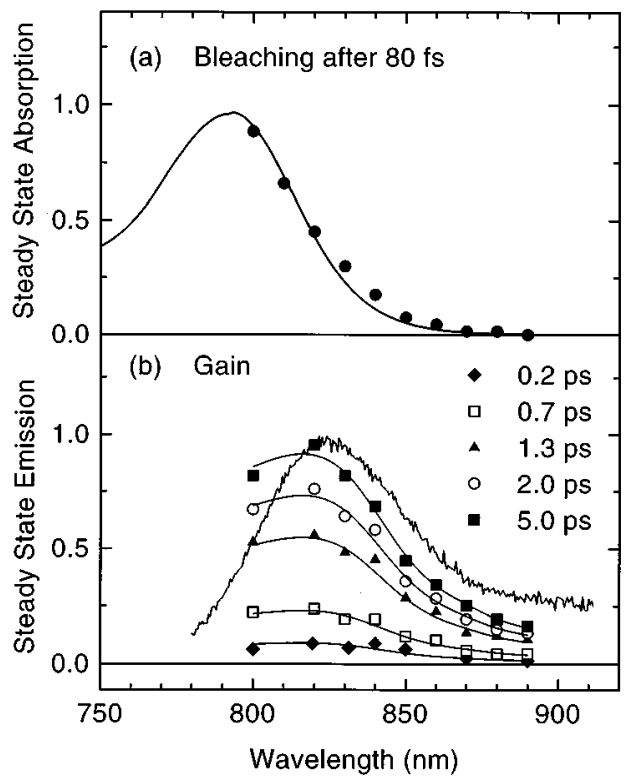

FIG. 6. (a) Steady state absorption spectrum (solid line) of IR 125. The solid circles show the ground state bleaching observed $80 \mathrm{fs}$ after the pump pulse [cf. Fig. 3(a)]. (b) Steady state (solid experimental line extending from 780 to $910 \mathrm{~nm}$ ) and transient emission spectra of IR 125. The transient spectra were derived from the spectrally and temporally resolved measurements on the picosecond time scale [cf. Fig. 3(b)] and reveal the buildup of stimulated emission from the $S_{1}$ state. Smooth solid lines are given for each time delay as a guide to the eye. The blueshift of the band for $5 \mathrm{ps}$ (solid squares) compared to the steady state spectrum is due to the incomplete solvation of the excited molecules in the polar solvent ethylene glycol, occurring on a slower time scale.

to the eye. For comparison, the $S_{0}-S_{1}$ absorption band [solid line in Fig. 6(a)] and the spectrum of stimulated emission [solid experimental line in Fig. 6(b)] are shown. The latter was calculated from the experimentally observed spontaneous emission spectrum. The transient gain spectra follow essentially the steady state emission spectrum. However, there is a blue shift of approximately $10 \mathrm{~nm}$ compared to the steady state emission band. This result is related to the relatively slow solvation of the excited molecules in the polar solvent ethylene glycol as will be discussed below.

\section{DISCUSSION}

The experimental results are now discussed by analyzing the time-resolved data on the different time scales separately. For the two-color experiment the transient signal consists of several components: (1) coherent oscillatory contributions around delay zero $\left(-50 \mathrm{fs}<t_{\text {delay }}<+50 \mathrm{fs}\right)$ and much weaker coherent oscillations at later times, (2) bleaching due to the depletion of the electronic ground state $S_{0}$, (3) stimulated emission after accumulation of excited molecules at the bottom of the $S_{1}$ state, (4) solvation in ethylene glycol, and (5) fluorescence decay of the $S_{1}$ state. The transient signal of the one-color experiment displays a very short spike around zero delay and steplike ground state bleaching.

\section{A. Coherent contributions}

Both the one-color transient recorded with probe pulses at $425 \mathrm{~nm}$ (upper trace in Fig. 2) and the two-color pumpprobe data (Fig. 3) exhibit coherent features around delay zero. At some spectral positions, oscillations persist into the picosecond regime.

(i) One-color experiment at $425 \mathrm{~nm}$ : The pulse-limited transmission peak in the one-color data is present for parallel polarizations of pump and probe but decreases substantially for orthogonal polarizations. This points to the coherent character of this signal which is dominated by the nonlinear coherent coupling of pump and probe pulses. Degenerate fourwave-mixing (DFWM) in the geometry of slightly crossed beams makes the strongest contribution to this signal. Within the electronic dephasing time of the $S_{0}-S_{n}$ transition, a transient grating is formed by pump and probe pulses in second order through which part of the pump wave is diffracted into the probe direction and enhances the probe intensity. In addition, there may be coherent contributions due to a pump interaction perturbed free induction decay (PFID) of the coherent polarization created by the probe wave. ${ }^{24}$ In the case of a small detuning with respect to the pure electronic transition, these terms lead to a transmission peak and oscillations around delay zero (referred to as detuning oscillations in Refs. 25-27).

(ii) Two-color experiment: The very fast oscillatory feature in the spectrally resolved data (left hand side of Fig. 3) is due to the coherent motion of a vibrational wave packet in the electronic ground state $S_{0}$ that is excited by the pump pulse via resonant impulsive stimulated Raman scattering (RISRS). ${ }^{10,20} \mathrm{~A}$ first interaction of the electric field of the pump pulse with the $S_{0}-S_{n}$ transition creates a coherent electronic polarization between the two potential surfaces (but no population change). In terms of wave packet dynamics, this is equivalent to a projection of a vibrational wave packet consisting of the modes which couple to the electronic transition from the $S_{0}$ to the $S_{n}$ potential surface. ${ }^{10,28}$ There, it propagates according to the vibrational Hamiltonian of the respective electronic state $S_{n}$ while the coherent $S_{0}-S_{n}$ excitation is damped with the electronic dephasing time $T_{2, \mathrm{el}} \approx 10-60 \mathrm{fs}$. Within $T_{2, \mathrm{el}}$, a second interaction of the pump pulse with coherently excited molecules projects the displaced vibrational wave packet back to the potential surface of the electronic ground state creating a coherent vibrational motion in $S_{0}$. Effective impulsive Raman excitation is made possible by the large bandwidth of pump pulses (700 $\mathrm{cm}^{-1}$ ). The vibrational modes most efficiently excited by an impulsive Raman process have frequencies within this 700 $\mathrm{cm}^{-1}$ range.

The multidimensional vibrational wave packet propagating in the $S_{0}$ state broadens rapidly and is eventually damped by vibrational dephasing of the individual modes with time constants $T_{2 \text {,vib }}$ of 0.2 to 2 ps. The spreading of the wave packet on a $50 \mathrm{fs}$ time scale is due to (i) the different time evolution of the modes involved according to their vibrational frequencies, and (ii) to initial phase shifts that result from the different shifts of the excited state potential minima 
along the different vibrational modes. The wave packet represents a nonstationary perturbation of the ground state vibrational wave function ${ }^{10,29}$ which is visualized via the probe pulses resonant to the $S_{0}-S_{1}$ transition. Mediated by the coordinate dependent distance between the $S_{1}$ and $S_{0}$ potential surfaces, the evolution of this nonstationary perturbation is mapped out by the various frequency components of the probe pulses [Fig. 3(a)]. The bottom of the $S_{0}$ potential surface is monitored by the short-wave edge of the probe bandwidth whereas the longwave edge is due to transitions in the displaced wave packet region. This causes an enhanced transmission from 800 to $820 \mathrm{~nm}$ and a pronounced induced absorption from 840 to $890 \mathrm{~nm}$ as shown in Fig. 3(a).

The initial wave packet dynamics induced by RISRS can be controlled by a chirp on the pump pulses. As shown in Figs. 4(a) and 4(b), we observe a distinct dependence of the peaklike signal around delay zero on the chirp of the pump pulses. In comparison to the curves with negligible chirp (solid lines), data recorded with pump pulses of negative chirp [open circles in Fig. 4(b)] exhibit a stronger coherent Raman-type excitation than the data with opposite chirp of the same magnitude [solid circles in Fig. 4(a)]. This behavior can be easily understood in terms of wave packet dynamics. The excited wave packet on the $S_{n}$ potential surface propagates along the vibrational coordinates, in general, from regions of larger $S_{n}-S_{0}$ separation to a region with a smaller separation. Consequently, excitation pulses with negative chirp couple coherently to the $S_{n}-S_{0}$ transition longer than pulses of opposite chirp. The longer interaction time leads to a tighter focusing of the wave packet in the electronic ground state which is tantamount to a more pronounced coherent Raman-type excitation. A similar result was reported recently for one-color experiments on the dye LD690. ${ }^{30}$

Additional recurrences of wave packet propagation at later delay times are strongly damped in the time-resolved data due to the rapid spreading discussed above. Nevertheless, the spectrally and time resolved data in Figs. 5(a) to 5(c) which were recorded at the spectral edges of the probe pulses, show an oscillatory behavior on a longer time scale. As described in Ref. 31, the observed phenomenon is due to an amplification effect of oscillations in the nonlinear third order polarization at the edges of both the probe spectrum and the $S_{0}-S_{1}$ absorption spectrum. By subtracting the simulated curves of the picosecond kinetics, we extracted the oscillatory traces which are shown as solid curves below the original data on the left-hand side of Figs. 5(a) and (b). The Fourier transforms of these oscillations which are displayed on the right-hand side of Fig. 5, reveal strong resonances at low frequencies of 50 and $170 \mathrm{~cm}^{-1}$. These resonances are due to vibrational modes of the molecule or of the moleculesolvent system with particularly long dephasing times ( $T_{2}^{\text {vib }} \cong 0.5$ to $2 \mathrm{ps}$ ).

In principle, the vibrational modes coupling to the $S_{0}-S_{n}$ and the $S_{0}-S_{1}$ transitions can be identified by resonance Raman spectroscopy at the respective spectral positions. Suppression of a strong background due to fluorescence from the dye molecules and/or impurities in the sample were the main experimental problems in recording

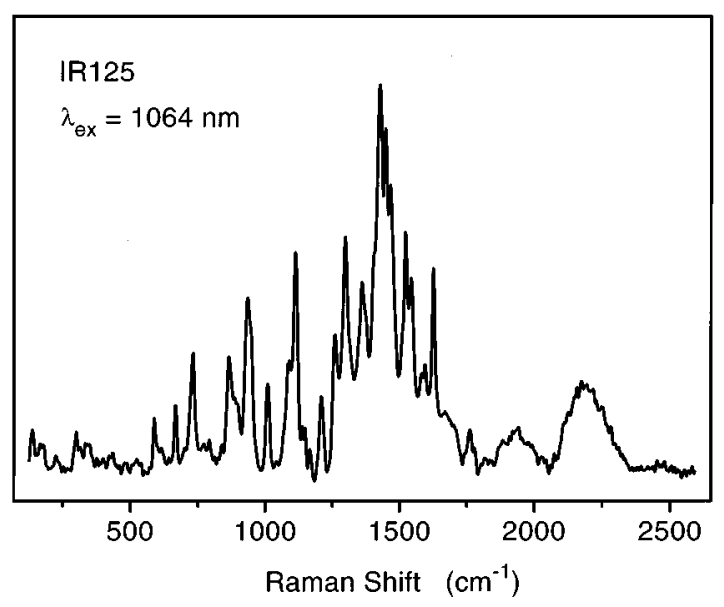

FIG. 7. Raman spectrum of a $10^{-3} \mathrm{M}$ solution of IR 125 in ethylene glycol after excitation with the $1064 \mathrm{~nm}$ light of a cw Nd:YAG laser. The raw Raman spectrum contained large contributions from solvent scattering and dye fluorescence. These were subtracted to obtain the spectrum shown.

such spectra. We therefore recorded Raman data under nonresonant conditions. Figure 7 shows the Raman spectrum of a $10^{-3} \mathrm{M}$ solution of IR125 in ethylene glycol after excitation with a continuous-wave Nd:YAG laser $(\lambda=1064 \mathrm{~nm})$. The raw spectrum contains contributions from both the dye fluorescence and the Raman scattering of the solvent. By comparison of spectra measured for various dye concentrations, the Raman spectrum of the dye molecules shown in Fig. 7 was deduced. About 20 lines of comparable strength are found in the frequency range from 600 to $1700 \mathrm{~cm}^{-1}$. The spectrum of Fig. 7 is close to the Raman spectrum of IR125 adsorbed on graphite for fluorescence quenching. ${ }^{32}$ The latter was measured with excitation at $784 \mathrm{~nm}$, fully resonant to the $S_{0}-S_{1}$ transition. A second spectrum recorded with excitation at $514 \mathrm{~nm}$, i.e., preresonant to higher lying singlet states, contains a subset of about ten lines present in the Raman spectrum measured in resonance with the $S_{0}-S_{1}$ transition. We conclude from the Raman data that vibrational wave packets created by RISRS in the electronic ground state are made up of a relatively large number of medium to high frequency modes, leading to the rapid spreading on the ground state potential hypersurface as found in our time-resolved measurements. This situation is quite similar to the one found in previous picosecond work. ${ }^{33}$ In these experiments wave packets with contributions from many coupled vibronic eigenstates in anthracene were excited and the transient signals showed an initial decay with nearly pulse limited shape. Consequently, small oscillatory signals with an amplitude of less than $10 \%$ of the total signal were seen.

Recently, both time resolved pump-probe recordings 9 and resonance Raman spectra were reported for nile blue en $^{34}$ and malachite green. ${ }^{35}$ Nile blue shows persistent regular oscillations in the time trace with one single Fourier component at $586 \mathrm{~cm}^{-1}$. A mode at $590 \mathrm{~cm}^{-1}$ is found to dominate the resonance Raman spectrum. For malachite green a very irregular structure is found in the pump-probe trace that decreases to less than half of the maximum signal within 100 
fs. At the same time, the resonance Raman spectrum displays a multitude of lines similar to IR125. This comparison nicely supports the notions discussed above for the explanation of the fast damping of the oscillatory signal components.

It is important to note that our two-color experiments allow the selective study of coherent wave packet motion in the electronic ground state. This fact is in contrast to various other pump-probe experiments on the $10 \mathrm{fs}$ and longer time scales ${ }^{4,9}$ where the ultrashort pump and probe pulses are at a common wavelength and ground and excited state dynamics is not separable. Furthermore, the use of well-separated excitation and probe wavelengths reduces the number of contributions to the third-order optical response of the molecules significantly. As a result, effects like coherent detuning oscillations $^{25-27}$ can be completely neglected.

\section{B. Bleaching due to ground state depletion}

After delay times of about 100 fs the coherent contributions to the signals are strongly damped and subsequent transient signals are therefore governed by incoherent dynamics. The latter is due to molecules excited to vibronic $S_{n}$ levels. The time resolved pump probe data at $425 \mathrm{~nm}$ (upper trace in Fig. 2) show an essentially constant transmission increase for delay times $>100$ fs. The absence of any dynamics in the subpicosecond or picosecond regime gives evidence that the steplike transmission increase has to be attributed exclusively to ground state depletion. Ground state bleaching is also observed in the two-color experiment. The spectrum of this signal which is shown in Fig. 6(a) for a delay time of 80 fs (solid circles) has been determined quantitatively from the measured amplitude of the signal, the concentration of excited molecules, and the sample thickness. The error of the absolute values is about $\pm 20 \%$. By comparison the relative uncertainty of data at different spectral positions is negligible. The transient bleaching follows exactly the stationary absorption band of unexcited molecules (solid line). This very good agreement demonstrates that excited state absorption from $S_{1}$ to higher singlet levels is negligible in this spectral range. The bleaching is a constant contribution to the overall signal on the picosecond time scale and eventually decays with the $S_{1}$ lifetime of $460 \mathrm{ps}$.

\section{Stimulated emission from $S_{n}$ and evolution of the $S_{1}$ emission band}

(i) One-color experiment at $425 \mathrm{~nm}$ : A possible contribution of stimulated emission from excited electronic states $S_{n}$ to the pump probe signal (upper trace in Fig. 2) might only occur within the first 50 fs temporally overlapping with the coherent effects discussed above. In terms of wave packet dynamics on the excited potential surface, this process is equivalent to a fast movement and/or spreading of the excited wave packet away from the optically coupled Franck-Condon region of the $S_{0}-S_{n}$ transition. Any deviation of the ground and excited state potential surfaces from the idealized displaced harmonic oscillator model (i.e., linear electron-phonon coupling) leads-already during the excitation process - to a fast propagation of the multidimensional wave packet into other regions of the potential hypersurface. ${ }^{36}$ Furthermore, redistribution of vibrational excitation within the $S_{n}$ vibrational manifold which is caused by anharmonic interactions and internal conversion processes to other excited electronic states, deplete the optically coupled Franck-Condon region of the $S_{0}-S_{n}$ transition. Such processes quench the stimulated emission from the initially excited $S_{n}$ states on a time scale shorter than about 50 fs and suppress recurrences of the wave packet.

(ii) In the two-color experiment, the subsequent time evolution of the $S_{n}$ population is monitored via stimulated emission from vibronic $S_{1}$ states, showing a delayed rise with a time constant of 1.2 ps. The gain at the different spectral positions follows the same kinetics, as can be seen from the data in Fig. 3(b). The transient emission bands plotted in Fig. 6(b) were derived from the total transmission change at fixed delay time by subtracting the amplitude of ground state bleaching at a delay time of $80 \mathrm{fs}$ [Fig. 6(a)]. Within the experimental accuracy, the emission spectrum develops without shifting or changing its shape.

A number of relaxation steps are responsible for the delayed rise of stimulated emission from $S_{1}$ levels. First, population is transferred from the initially excited $S_{n}$ states to vibronic $S_{1}$ levels by internal conversion processes. This relaxation is governed by the nonadiabatic coupling between different excited electronic states and can proceed either by sequential steps $S_{n}-S_{n-1} \ldots . .-S_{1}$ or by direct coupling between the initially excited states and the $S_{1}$ manifold. Our data give no detailed insight into the different pathways. However, the fact that the initial $S_{n}$ population disappears much faster than the build-up of $S_{1}$ population demonstrates that the depopulation of the directly excited $S_{n}$ states is not the rate-limiting step in $S_{n}-S_{1}$ relaxation. Intramolecular redistribution towards a quasiequilibrium distribution of vibronic $S_{1}$ populations and energy transfer from the dye molecules to the surrounding solvent represent a second class of relaxation processes determining the onset of stimulated emission. The absence of any reshaping of the emission spectrum [cf. Fig. 6(b)] suggests that there is no significant nonequilibrium excitation of the vibrational modes coupling to the electronic $S_{1}-S_{0}$ transition.

To get some information on the electronic states possibly involved in the $S_{n}-S_{1}$ relaxation and on the position of the different electronic transitions, quantum-chemical calculations based on a ZINDO method were performed for IR125 and oxazine $1 .{ }^{37}$ For IR125, the photon energy of the excitation pulses is close to the $S_{7}$ level. The calculated spectra show five groups of absorption lines at 320, 370, 450, 500, and $750 \mathrm{~nm}$. This pattern is in qualitative agreement with the position of resonances in the absorption spectrum of Fig. 1.

In a sequential scheme for $S_{n}-S_{1}$ internal conversion, the $S_{2}-S_{1}$ relaxation represents the final step. For IR125, the calculation gives a large energy gap between the $S_{2}$ and $S_{1}$ state of about $5700 \mathrm{~cm}^{-1}$. In contrast, the calculated $S_{2}-S_{1}$ separation in oxazine 1 has a smaller value of $3000 \mathrm{~cm}^{-1}$. The time constants of $S_{1}$ population as determined from the onset of stimulated emission are 1.2 ps for IR125 and $200 \mathrm{fs}$ for oxazine $1 .{ }^{18}$ The pronounced decrease of the relaxation 
time for a smaller $S_{2}-S_{1}$ gap is in qualitative agreement with semiempirical energy gap laws ${ }^{38,39}$ which predict an exponential decrease of the relaxation time for smaller energy gaps. Thus the different $S_{n}-S_{1}$ relaxation rates of IR125 and oxazine 1 might be related to the rate-limiting $S_{2}-S_{1}$ internal conversion.

\section{Solvation in ethylene glycol}

A closer inspection of the transient emission spectra [Fig. 6(b)] points to an incomplete relaxation at a delay time of $5 \mathrm{ps}$. A comparison with the stationary spectrum of stimulated emission (solid line) shows that the final position of the emission spectrum is shifted by $10 \mathrm{~nm}$ towards longer wavelengths. This redshift is due to the polar solvation process in ethylene glycol. The rearrangement of the solvent molecules in response to the changed charge distribution (electronic wave function) of the solute after excitation leads to a lowering of the energy of the entire system, solute and solvent, resulting in a redshifted emission band. A value of $100 \mathrm{ps}$ was reported for the solvation time of ethylene glycol at room temperature, ${ }^{40}$ i.e., the solvation of IR 125 is not yet complete within the $10 \mathrm{ps}$ time window considered in our experiment.

\section{SUMMARY}

In conclusion, we investigated the coherent and incoherent dynamics of large dye molecules (IR 125) in solution on time scales from 20 fs to $5 \mathrm{ps}$ in temporally and spectrally resolved pump-probe experiments. The molecules are excited with $30 \mathrm{fs}$ pulses at $425 \mathrm{~nm}$ which couple the electronic ground state $S_{0}$ to higher lying singlet states $S_{n}(n \approx 7)$. With probe pulses of the same wavelength we observe, during pump-probe coincidence, a coherent peaklike response superimposed onto a steplike bleaching signal which is due to depletion of the electronic ground state remaining constant within the $S_{1}$ lifetime. Temporally and spectrally resolved experiments were also performed with 20 fs probe pulses resonant to both the $S_{1}-S_{0}$ absorption and emission band. In part of the molecules, excitation resonant with $S_{0}-S_{n}$ transitions induces a coherent vibrational excitation in the electronic ground state by a resonantly enhanced impulsive Raman process. The coherent ground state dynamics is monitored via fast oscillations of the $S_{1}-S_{0}$ absorption band. The coherently excited oscillations extend at some spectral positions into the picosecond time regime and were enhanced or weakened by chirping of the pump pulses. Molecules excited to $S_{n}$ levels show a steplike bleaching of the $S_{1}-S_{0}$ absorption due to depletion of the electronic ground state which exactly follows the stationary absorption band. After intramolecular $S_{n}-S_{1}$ relaxation, stimulated $S_{1}-S_{0}$ emission occurs with a rise time of 1.2 ps. This is much slower than the relaxation of the initially excited $S_{n}$ states. The corresponding emission band, which is monitored via transient gain spectra, develops within the first 5 ps without changing its shape. On a slower picosecond time scale, the emission undergoes a $10 \mathrm{~nm}$ redshift caused by solvation in the solvent ethylene glycol. The emission from the $S_{1}$ state decays with a lifetime of 460 ps.

\section{ACKNOWLEDGMENTS}

We would like to thank Dr. Michael Pfeiffer, Max-BornInstitut, for performing the ZINDO calculations and for valuable discussions, Dr. Klaus-Werner Brzezinka, Bundesanstalt für Materialforschung und -prüfung Berlin, for performing the Raman measurements and Michael Schulz, Max-BornInstitut, for performing the fluorescence decay measurement. S. H. Ashworth gratefully acknowledges support from an Alexander von Humboldt Stiftung fellowship.

${ }^{1}$ M. J. Rosker, F. W. Wise, and C. L. Tang, Phys. Rev. Lett. 57, 321 (1986).

${ }^{2}$ C. H. Brito Cruz, R. L. Fork, W. H. Knox, and C. V. Shank, Chem. Phys. Lett. 132, 341 (1986).

${ }^{3}$ E. T. J. Nibbering, D. A. Wiersma, and K. Duppen, Phys. Rev. Lett. 66, 2464 (1991).

${ }^{4}$ T. Joo, and A. C. Albrecht, Chem. Phys. 173, 17 (1993).

${ }^{5}$ M. S. Pshenichnikov, K. Duppen, and D. W. Wiersma, Phys. Rev. Lett. 74, 674 (1995).

${ }^{6}$ S. Mukamel, Annu. Rev. Phys. Chem. 41, 647 (1990).

${ }^{7}$ W. P. de Boeij, M. S. Pshenichnikov, and D. A. Wiersma, Chem. Phys. Lett. 238, 1 (1995).

${ }^{8}$ K. A. Nelson, and E. P. Ippen, Adv. Chem. Phys. 75, 1 (1989).

${ }^{9}$ H. L. Fragnito, J.-Y. Bigot, P. C. Becker, and C. V. Shank, Chem. Phys. Lett. 160, 101 (1989).

${ }^{10}$ W. T. Pollard, S.-Y. Lee, and R. A. Mathies, J. Chem. Phys. 92, 4012 (1990).

${ }^{11}$ P. Vöhringer, R. A. Westervelt, T.-S. Yang, D. C. Arnett, M. J. Feldstein, and N. F. Scherer, J. Raman Spectrosc. 26, 535 (1995).

${ }^{12}$ For a review see, T. Elsaesser, and W. Kaiser, Annu. Rev. Phys. Chem. 42, 83 (1991).

${ }^{13}$ A. M. Weiner, and E. P. Ippen, Chem. Phys. Lett. 114, 456 (1985).

${ }^{14}$ A. Mokhtari, A. Chebira, and J. Chesnoy, J. Opt. Soc. Am. B 7, 1551 (1990).

${ }^{15}$ M. Kasha, Discuss. Faraday Soc. 9, 14 (1950).

${ }^{16}$ For a review see, K. F. Freed, in Radiationless Processes in Molecules and Condensed Phases, Topics in Applied Physics Vol. 15, edited by F. K. Fong (Springer Berlin, 1976), p. 23.

${ }^{17}$ C. V. Shank, E. P. Ippen, and O. Teschke, Chem. Phys. Lett. 45, 291 (1977).

${ }^{18}$ F. Laermer, T. Elsaesser, and W. Kaiser, Chem. Phys. Lett. 156, 381 (1989).

${ }^{19}$ F. Laermer, W. Israel, and T. Elsaesser, J. Opt. Soc. Am. B 7, 1604 (1990).

${ }^{20}$ T. Hasche, S. H. Ashworth, E. Riedle, M. Woerner, and T. Elsaesser, Chem. Phys. Lett. 244, 164 (1995).

${ }^{21}$ P. F. Curley, Ch. Spielmann, T. Brabec, F. Krausz, E. Wintner, and A. J. Schmidt, Opt. Lett. 18, 54 (1993).

${ }^{22}$ M. T. Asaki, C.-P. Huang, D. Garvey, J. Zhou, H. C. Kapteyn, and M. M. Murnane, Opt. Lett. 18, 977 (1993).

${ }^{23}$ S. H. Ashworth, M. Joschko, M. Woerner, E. Riedle, and T. Elsaesser, Opt. Lett. 20, 2120 (1995).

${ }^{24}$ C. H. Brito Cruz, J. P. Gordon, P. C. Becker, R. L. Fork, and C. V. Shank, IEEE J. Quant. Electron. 24, 261 (1988).

${ }^{25}$ P. Cong, H. P. Druel, and J. D. Simon, Chem. Phys. Lett. 212, 367 (1993).

${ }^{26}$ M. Chachisvilis, H. Fidder, and V. Sundström, Chem. Phys. Lett. 234, 141 (1995).

${ }^{27}$ E. Gaizauskas, and L. Valkunas, Opt. Commun. 109, 75 (1994).

${ }^{28}$ S.-Y. Lee and E. J. Heller, J. Chem. Phys. 71, 4777 (1979).

${ }^{29}$ U. Banin, A. Bartana, S. Ruhman, and R. Kosloff, J. Chem. Phys. 101, 8461 (1994).

${ }^{30}$ C. J. Bardeen, Q. Wang, and C. V. Shank, Phys. Rev. Lett. 75, 3410 (1995).

${ }^{31}$ W. T. Pollard, S. L. Dexheimer, Q. Wang, L. A. Peteanu, C. V. Shank, and R. A. Mathies, J. Phys. Chem. 96, 6147 (1992). 
${ }^{32}$ M. R. Kagan and R. L. McCreery, Anal. Chem. 66, 4159 (1994).

${ }^{33}$ P. M. Felker and A. H. Zewail, in Advances in Chemical Physics (Wiley, New York, 1988), Vol. LXX, p. 265.

${ }^{34}$ M. K. Lawless and R. A. Mathies, J. Chem. Phys. 96, 8037 (1992).

${ }^{35}$ H. B. Lueck, D. C. Daniel, and J. L. McHale, J. Raman. Spectrosc. 24, 363 (1993).
${ }^{36}$ V. Hizhnyakov and I. Tehver, Phys. Stat. Sol. 21, 755 (1967).

${ }^{37}$ M. Pfeiffer (unpublished).

${ }^{38}$ R. Englman and J. Jortner, Mol. Phys. 18, 145 (1970).

${ }^{39}$ S. Murata, C. Iwanaga, T. Toda, and H. Kokobun, Ber. Bunsenges. Phys. Chem. 76, 1176 (1972).

${ }^{40}$ J. D. Simon, Acc. Chem. Res. 21, 128 (1988). 